\title{
Exact Solution of Nonlinear Partial Differential Equations, Using the New Tan-Cot Function Method
}

\author{
P. N. Habu'; F. Attah ${ }^{2}$ \\ ${ }^{1}$ Department of Mathematics, Federal University Lafia, Nigeria. \\ ${ }^{2}$ Department of Mathematics, Federal University Lafia, Nigeria.
}

Abstract: A traveling wave solution, using the proposed Tan-Cot Function Method for Nonlinear Partial Differential Equations, has been established in this paper. This method is used to obtain new solitary wave solutions for nonlinear partial differential equations such as the Coupled Klein-Gordon system of equations, Huxley equation, Dispersive Long Wave Equation and Korteweg-dVries (KDV) equation.

\section{Introduction}

Nonlinear partial differential equations govern many physical, biological, chemical and other area of study. One of the most exciting advances of nonlinear science and theoretical physics has been the development of methods to look for exact solutions of nonlinear partial differential equations as discussed by Mahmood Jawal Abdul Rasool Abu Al-Shaeer[4]. Nonlinear partial differential equations can provide much physical information and more insight into the physical aspects of problems, and therefore can lead to further applications

In particular, nonlinear wave phenomena of dispersion, dissipation, diffusion, reaction and convection are very important in nonlinear wave equation as discussed by Mahmood Jawal Abdul Rasool Abu Al-Shaeer [4].

As discussed by Mahmood Jawal Abdul Rasool Abu Al Shaeer[4], he used the Tan-Cot function method to establish solitary wave exact solutions for the $(1+1)$-dimensional Ito equation of Pochhamner-Chree (PC) equation, MIKP equation, Konopelchenko and Dubrovsky (KD) system of equations.

Also, as discussed by Anwar Ja'far Mohammed Jawal [1] used the Tan-Cot function method to solve the (2+1)-dimensional nonlinear Schrodinger equation, Gardner equation, modified KDV equation, Perturbed Burger Benjamine-Bona Mahony (BBM) equation.

The Coupled Klein-Gardon equation as discussed by Nasir Taghizadeh, Mohammad Mirzaza and Faroozon Farahrooz [5], using the infinite series method and exact solition solutions were obtained for the Huxley equation as discussed by N. Taghizadeh et al [6], by applying the modified $\left(\frac{G^{\prime}}{G}\right)$-expansion method. Anwar Ja'afar Mohammad Jaw[1] used the tanh and sine-cosine methods to obtain exact solition solutions for the nonlinear dispersive long wave equation.

In this paper, we applied the new Tan-Cot function method to solve the Klein-Gordon system of equations, Huxley equation, nonlinear Dispersive Long Wave equation and Korteweg-dVries equation, all now given respectively by:

$$
\begin{aligned}
& \mathrm{u}_{\mathrm{xx}}-\mathrm{u}_{\mathrm{tt}}-\mathrm{u}+2 \mathrm{u}^{3}+2 \mathrm{uv}=0 \\
& v_{x}-v_{t}-4 u u_{t}=0 \\
& \mathrm{u}_{\mathrm{t}}=\mathrm{u}_{\mathrm{xx}}+\mathrm{u}(\mathrm{k}-\mathrm{u})(\mathrm{u}-1) \text {. } \\
& \mathrm{u}_{\mathrm{yt}}+\mathrm{v}_{\mathrm{xx}}+\frac{1}{2}\left(\mathrm{u}^{2}\right)_{\mathrm{xy}}=0 \\
& v_{\mathrm{t}}+\left(\mathrm{uv}+\mathrm{u}+\mathrm{u}_{\mathrm{xy}}\right)_{\mathrm{x}}=0 \\
& \Psi_{\mathrm{t}}+\Psi \Psi_{\mathrm{x}}+\Psi_{\mathrm{xxx}}=0
\end{aligned}
$$

\subsection{The Tan-Cot Function Method}

\section{Materials and Methods}

A nonlinear partial differential equations in the form as discussed by Mahmood Jawal Abdul Rasool Abu Al-Shaeer [4]

$\mathrm{F}\left(\mathrm{u}, \mathrm{u}_{\mathrm{t}}, \mathrm{u}_{\mathrm{x}}, \mathrm{u}_{\mathrm{y}}, \mathrm{u}_{\mathrm{tt}}, \mathrm{u}_{\mathrm{xx}}, \mathrm{u}_{\mathrm{yy}}, \mathrm{u}_{\mathrm{xy}}, \ldots\right)=0$

where $\mathrm{u}(\mathrm{x}, \mathrm{y}, \mathrm{t})$ is a traveling wave solution of the nonlinear partial differential equations given in (5). We use the transformations,

$\mathrm{u}(\mathrm{x}, \mathrm{y}, \mathrm{t})=\mathrm{f}(\xi)$,

Where $\xi=\mathrm{kx}+\partial \mathrm{y}-\lambda \mathrm{t}$ 
This enables us to use the following changes:

$\frac{\partial \mathrm{f}}{\partial \mathrm{t}}=-\lambda \frac{\mathrm{df}}{\mathrm{d} \xi}, \frac{\partial \mathrm{f}}{\partial \mathrm{x}}=-\mathrm{k} \frac{\mathrm{df}}{\mathrm{d} \xi}, \frac{\partial \mathrm{f}}{\partial \mathrm{y}}=-\delta \frac{\mathrm{df}}{\mathrm{d} \xi}$

Using (8) to transform the nonlinear partial differential equation (5) to an ordinary

differential equation,

$\mathrm{Q}\left(\mathrm{f}, \mathrm{f}^{\prime}, \mathrm{f}^{\prime \prime}, \mathrm{f}^{\prime \prime \prime}, \ldots\right)=0$

The ordinary differential equation (9) is then integrated as long as all terms contain derivatives, where we neglect the integration constants. The solution of many nonlinear equations can be represented or expressed in the form [4],

$\begin{aligned} f(\xi) & =\alpha \tan ^{\beta}(\mu \xi),|\xi| \leq \frac{\pi}{2 \mu} \\ f(\xi) & =\alpha \operatorname{Cot}^{\beta}(\mu \xi),|\xi| \leq \frac{\pi}{2 \mu}\end{aligned}$

Where $\alpha, \mu$ and $\beta$ are paran

Where $\alpha, \mu$ and $\beta$ are parameters to be determined, $\alpha$ and $\mu$ are the wave number and wave speed respectively.

Accordingly, we now use,

$\mathrm{f}(\xi)=\alpha \tan ^{\beta}(\mu \xi)$

$\mathrm{f}^{\prime}=\alpha \beta \mu\left[\tan ^{\beta-1}(\mu \xi)+\tan ^{\beta+1}(\mu \xi)\right]$

$f^{\prime \prime}=\alpha \beta \mu^{2}\left[(\beta-1) \tan ^{\beta-2}(\mu \xi)+2 \beta \tan ^{\beta}(\mu \xi)+(\beta+1) \tan ^{\beta+2}(\mu \xi)\right]$

$\mathrm{f}^{\prime \prime \prime}=\alpha \beta \mu^{3}\left[(\beta-1)(\beta-2) \tan ^{\beta-3}(\mu \xi)+\left(3 \beta^{2}-3 \beta+2\right) \tan ^{\beta-1}(\mu \xi)+(\beta+1)(\beta+2) \tan ^{\beta}(\mu \xi)+\right.$

$2 \beta 2 \tan \beta+1 \mu \xi+\beta+1 \beta+2 \tan \beta+2 \mu \xi$

Let the expressions of $(\xi), \mathrm{f}^{\prime}, \mathrm{f}^{\prime \prime}$, and $\mathrm{f}^{\prime \prime \prime}$ constitute our equation 12 , or we may choose to use

$\mathrm{f}(\xi)=\alpha \operatorname{Cot}^{\beta}(\mu \xi)$

$\mathrm{f}^{\prime}=-\alpha \beta \mu\left[\operatorname{Cot}^{\beta-1}(\mu \xi)+\operatorname{Cot}^{\beta+1}(\mu \xi)\right]$

$f^{\prime \prime}=\alpha \beta \mu^{2}\left[(\beta-1) \tan ^{\beta-2}(\mu \xi)+2 \beta \operatorname{Cot}^{\beta}(\mu \xi)+(\beta+1) \operatorname{Cot}^{\beta+1}(\mu \xi)\right]$,

and so on. Again let the expression of $f(\xi), f^{\prime}, f^{\prime \prime}$ and so on for the tan and Cot be equation (12) and (13) respectively.

We now substitute equation (12) or (13) into equation (9), balance the tan and Cot functions and solve the resulting system of algebraic equations.

Next we collect all terms with the same power in

$\tan ^{\mathrm{k}}(\mu \xi)$ or $\operatorname{Cot}^{\mathrm{k}}(\mu \xi)$ and set to zero their coefficients to get a system of algebraic equations with the unknowns $\alpha, \beta$ and $\mu$, and solve the subsequent system of equations.

2.1.1 Applichation of the Tan-Cot Function Method to solve different Nonlinear Partial Differential Equations

2.1.1.1: The Coupled Klein-Gordon System of Equations as discussed Elsewhere

$[5,7]$.

It is as follows:

$\mathrm{u}_{\mathrm{xx}}-\mathrm{u}_{\mathrm{tt}}-\mathrm{u}+2 \mathrm{u}^{3}+2 \mathrm{uv}=0$

$$
v_{\mathrm{x}}-\mathrm{v}_{\mathrm{t}}-4 \mathrm{uu}_{\mathrm{t}}=0
$$

We use the transformations

$u(x, t)=u(\xi), v(x, t)=v(\xi), \xi=x-\lambda t$

and from equation (8), we have equation (14) transformed into the ordinary differential equations $u(\xi)$ and $v(\xi)$ $\left(1-\lambda^{2}\right) u^{\prime \prime}-u(\xi)+2 u^{3}(\xi)+2 u(\xi) v(\xi)=0$

By integrating the second equation in (16) with respect to $\xi$ and neglecting the constant of integration, we have

$v(\xi)=\frac{-2 \lambda}{1+\lambda} u^{2}(\xi)$

Substituting (17) into the first equation of (16) and integrating the resulting equation, we have

$\left(1-\lambda^{2}\right) u^{\prime \prime}(\xi)-u(\xi)+2 \frac{(1-\lambda)}{1+\lambda} u^{3}(\xi)=0$ 
which is an ordinary differential equation. Now to solve (18), we choose to use $\mathrm{u}=\alpha \tan ^{\beta}(\mu \xi)$, where

$\mathrm{u}^{\prime}=\alpha \beta \mu\left[\tan ^{\beta-1}(\mu \xi)+\tan ^{\beta+1}(\mu \xi)\right]$

$\mathrm{u}^{\prime \prime}=\alpha \beta \mathrm{u}^{2}\left[(\beta-1) \tan ^{\beta-2}(\mu \xi)+2 \beta \tan ^{\beta}(\mu \xi)+(\beta+1) \tan ^{\beta+2}(\mu \xi)\right]$.

where the expression for $u, u^{\prime}$ and $u^{\prime \prime}$ constitute equation (19). Substituting (19) into (18), we have $\left(1+\lambda^{2}\right) \alpha \beta u^{2}\left[(\beta-1) \tan ^{\beta-2}(\mu \xi)+2 \beta \tan ^{\beta}(\mu \xi)+(\beta+1) \tan ^{\beta+2}(\mu \xi)-\alpha \tan ^{\beta}(\mu \xi)+2 \frac{(1-\lambda)}{1+\lambda} \alpha^{3} \tan ^{3 \beta}(\mu \xi)=\right.$ 0

Equating exponents of each pair in equation (20), we have

$\beta+2=3 \beta, \Rightarrow \beta=1$

Substituting $\beta=1$ into equation (20) and simplify, then we get

$\left[2\left(1-\lambda^{2}\right) \alpha u^{2}-\alpha\right] \tan (\mu \xi)+\left[2\left(1-\lambda^{2}\right) \alpha u^{2}+2 \frac{(1-\lambda) \alpha^{3}}{1+\lambda}\right] \tan ^{3}(\mu \xi)=0$

Equating the coefficient of a pair of $\tan ^{\mathrm{k}}(\mu \xi)$ to zero in $(21)$, we have for $\tan (\mu \xi)$,

$2\left(1-\lambda^{2}\right) \alpha u^{2}-\alpha=0$

$\Rightarrow \mu= \pm \frac{1}{\sqrt{2}\left(1-\lambda^{2}\right)^{\frac{1}{2}}}$

and for $\tan ^{3}(\mu \xi), 2\left(1-\lambda^{2}\right) \alpha u^{2}+2 \frac{(1-\lambda) \alpha^{3}}{1+\lambda}=0$

$\alpha= \pm \frac{i(1+\lambda)^{\frac{1}{2}}}{\sqrt{2}\left(1-\lambda^{2}\right)^{\frac{1}{2}}}$

$\beta=1, \mu= \pm \frac{1}{\sqrt{2}\left(1-\lambda^{2}\right)^{\frac{1}{2}}}$ and $\alpha= \pm \frac{\mathrm{i}(1+\lambda)^{\frac{1}{2}}}{\sqrt{2}\left(1-\lambda^{2}\right)^{\frac{1}{2}}}$ into (17) and (19) we have

$\mathrm{u}(\mathrm{x}, \mathrm{t})= \pm \frac{\mathrm{i}(1+\lambda)^{\frac{1}{2}}}{\sqrt{2}\left(1-\lambda^{2}\right)^{\frac{1}{2}}} \tan \left( \pm \frac{1}{\sqrt{2}\left(1-\lambda^{2}\right)^{\frac{1}{2}}} \xi\right)$

$u(x, t)=i(1+\lambda)^{\frac{1}{2}} \tan \left( \pm \frac{1}{\sqrt{2}\left(1-\lambda^{2}\right)^{\frac{1}{2}}}(x-\lambda t)\right)$

2.1.1.2 The Huxley Equation as discussed by N. Taghizadeh et al [6]

We now consider the Huxley equation,

$\mathrm{u}_{\mathrm{t}}=\mathrm{u}_{\mathrm{xx}}+\mathrm{u}(\mathrm{k}-\mathrm{u})(\mathrm{u}-1)$....

where $\mathrm{k} \neq 0$ and $\mathrm{u}=\mathrm{u}(\mathrm{x}, \mathrm{t})$ is the solution of equation (26). We use the transformations

$\mathrm{u}(\mathrm{x}, \mathrm{t})=\mathrm{f}(\xi)$, Where $\xi=\mathrm{x}-\lambda \mathrm{t}$ and equation (8) to transform equation (26) to the ordinary differential equation $-\lambda u^{\prime}-u^{\prime \prime}+u^{3}-(k+1) u^{2}+k u=0$

The solution of (27) can be expressed as

$\mathrm{u}(\xi)=\alpha \tan ^{\beta}(\mu \xi)$, where

$\mathrm{u}^{\prime}=\alpha \beta \mu\left[\tan ^{\beta-1}(\mu \xi)+\tan ^{\beta+1}(\mu \xi)\right]$

$\mathrm{u}^{\prime \prime}=\alpha \beta \mathrm{u}^{2}\left[(\beta-1) \tan ^{\beta-2}(\mu \xi)+2 \beta \tan ^{\beta}(\mu \xi)+(\beta+1) \tan ^{\beta+2}(\mu \xi)\right]$,

and we represent the expressions of $u(\xi), u^{\prime}$ and $u^{\prime \prime}$ by equation (28). Substitute equation (28) into (27), we have, after simplification

$\left[\mathrm{k} \alpha-2 \alpha \beta^{2} \mathrm{u}^{2}\right] \tan ^{\beta}(\mu \xi)-\lambda \alpha \beta \mu \tan ^{\beta-1}(\mu \xi)-\alpha \beta \mathrm{u}^{2}(\beta-1) \tan ^{\beta-2}(\mu \xi)-\lambda \alpha \beta \mu \tan ^{\beta-1}(\mu \xi)-\alpha \beta \mathrm{u}^{2}(\beta+$ $1 \tan \beta+2 \mu \xi-\mathrm{k}+1 \alpha 2 \tan 2 \beta \mu \xi+\alpha 3 \tan 3 \beta \mu \xi=0$

Equating the exponents of each pair of the tan function in equation (29) we obtain

$\beta+2=2 \beta, \Rightarrow \beta=2$

Substituting $\beta=2$ into equation (29) and simplify, we have

$\left.\mathrm{k} \alpha-8 \alpha \mu^{2}\right] \tan ^{2}(\mu \xi)+4 \lambda \alpha \mu \tan (\mu \xi)-2 \alpha \mu^{2}-6 \alpha \mu^{2} \tan ^{4}(\mu \xi)-(\mathrm{k}+1) \alpha^{2} \tan ^{4}(\mu \xi)+\alpha^{3} \tan ^{6}(\mu \xi)=$ 0

In equation (30), equating the coefficients of each pair of the tan function, we get for

$\tan ^{2}(\mu \xi), \mathrm{k}-8 \mu^{2}=0 \Rightarrow \mu= \pm \sqrt{\frac{\mathrm{k}}{8}}$ 
and for $\tan ^{4}(\mu \xi),-\left(6 \alpha \mu^{2}+(k+1) \alpha^{2}\right)=0$

$\Rightarrow \alpha= \pm \frac{-3 \mathrm{k}}{4(\mathrm{k}+1)}$

Substituting $\mu= \pm \sqrt{\frac{\mathrm{k}}{8}}$ and $\alpha= \pm \frac{-3 \mathrm{k}}{4(\mathrm{k}+1)}$ into

$\mathrm{u}(\xi)=\alpha \tan ^{2}(\mu \xi)$, we get

$\mathrm{u}(\xi)=\mathrm{u}(\mathrm{u}, \mathrm{t})=\frac{-3 \mathrm{k}}{4(\mathrm{k}+1)} \tan ^{2}\left(\sqrt{\frac{\mathrm{k}}{8}}(\mathrm{x}-\lambda \mathrm{t})\right)$

2.1.1.3 The Dispersive Long Wave System of Equations as discussed by Anwar Ja'afar Mohammed Jawal [2]

The system of equation

$\mathrm{u}_{\mathrm{yt}}+\mathrm{v}_{\mathrm{xx}}+\frac{1}{2}\left(\mathrm{u}^{2}\right)_{\mathrm{xy}}=0$

$\mathrm{v}_{\mathrm{t}}+\left(\mathrm{uv}+\mathrm{u}+\mathrm{u}_{\mathrm{xy}}\right)_{\mathrm{x}}=0$

Now, using the traveling wave transformations

$\mathrm{u}(\mathrm{x}, \mathrm{y}, \mathrm{t})=\mathrm{u}(\xi), \mathrm{v}(\mathrm{x}, \mathrm{y}, \mathrm{t})=\mathrm{v}(\xi), \xi=\mathrm{kx}+\partial \mathrm{y}+\lambda \mathrm{t}$

and also using equation (8), the nonlinear system of partial differential equation (32) is

transformed to the system of ordinary differential equations

$$
\begin{gathered}
\delta u^{\prime \prime}(\xi)+k^{2} v^{\prime \prime}+k \delta\left(u u^{\prime}\right)^{\prime}=0 \\
\lambda v^{\prime}+k\left(u u^{\prime}\right)+k^{\prime} \delta u^{\prime \prime \prime}=0
\end{gathered}
$$

By integrating the first equation in (34) twice with zero constant, then it reduce to

$\delta \lambda u+k^{2} v+\frac{k \delta}{2} u^{2}=0$

Secondly, integrate the second equation of (34) once with zero constant, we have $\lambda v+k u v+k u+k^{2} \delta u^{\prime \prime}=0$

From equation (35), $v=\frac{\mathrm{k}^{2} \delta \mathrm{u}^{2}}{2 \mathrm{k}^{2}}-\frac{\delta \lambda \mathrm{u}}{\mathrm{k}^{2}}$, substitute $\mathrm{v}$ into (35), and simplify, we get

$[-\lambda-\mathrm{ku}]\left[\frac{\mathrm{k}^{2} \delta \mathrm{u}^{2}+2 \delta \lambda \mathrm{u}}{2 \mathrm{k}^{2}}\right]+\mathrm{ku}+\mathrm{k}^{2} \delta \mathrm{u}^{\prime \prime}=0$

which is an ordinary differential equation. We now seek the solution of (37) as, $\mathrm{u}(\xi)=\alpha \operatorname{Cot}^{\beta}(\mu \xi)$, where

$$
u^{\prime \prime}=\alpha \beta u^{2}\left[(\beta-1) \operatorname{Cot}^{\beta-2}(\mu \xi)+2 \beta \operatorname{Cot}^{\beta}(\mu \xi)+(\beta+1) \operatorname{Cot}^{\beta+2}(\mu \xi)\right]
$$

and we represent the expressions of $u$ and $\mathrm{u}^{\prime \prime}$ by equation (38). Substitute equation (38) into (37) and simplify, we get

$\left[2 \beta^{2} \mathrm{u}^{2} \mathrm{k}^{2} \delta \alpha+\mathrm{k} \alpha-\frac{\delta \lambda^{2} \alpha}{\mathrm{k}^{2}}\right] \operatorname{Cot}^{\beta}(\mu \xi)-\left[\frac{\mathrm{k}^{2} \delta \alpha}{\mathrm{k}}-\frac{\alpha^{2} \delta \lambda}{\mathrm{k}}\right] \operatorname{Cot}^{2 \beta}(\mu \xi)+\mathrm{k}^{2} \delta \alpha \beta \mathrm{u}^{2}(\beta-1) \operatorname{Cot}^{\beta-2}(\mu \xi)+\mathrm{k}^{2} \delta \alpha(\beta+$ $1 \operatorname{Cot} \beta+2 \mu \xi-\alpha 3 \delta 2 \operatorname{Cot} 3 \beta \mu \xi=0$. .

In (39), equating the exponents of each pair of the Cot function, we get

$\beta+2=3 \beta, \Rightarrow \beta=1$

Substituting $\beta=1$ into equation (39) and simplify, we have

$\left[2 \mu^{2} \mathrm{k}^{2} \delta \alpha+\mathrm{k} \alpha-\frac{\delta \lambda^{2} \alpha}{2}\right] \operatorname{Cot}(\mu \xi)-3 \lambda \mathrm{k} \delta \alpha^{2} \operatorname{Cot}^{2}(\mu \xi)+2 \mu^{2} \mathrm{k}^{2} \delta \alpha \operatorname{Cot}^{2}(\mu \xi)-\frac{\alpha^{3} \delta}{2} \operatorname{Cot}^{3}(\mu \xi)=$

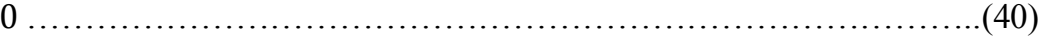

In equation (40), equating the coefficients of each pair of the $\operatorname{Cot}^{\mathrm{k}}(\mu \xi)$ to zero for each pair, we get for $\operatorname{Cot}(\mu \xi)$,

$2 \mu^{2} \mathrm{k}^{2} \delta \alpha+\mathrm{k} \alpha-\frac{\delta \lambda^{2} \alpha}{2}=0$, which gives

$\mu= \pm\left[\frac{\lambda^{2}}{2 \mathrm{k}^{4}}-\frac{1}{2 \mathrm{k} \delta}\right]^{\frac{1}{2}}$ and for

$\operatorname{Cot}^{2}(\mu \xi), 2 \mu^{2} \mathrm{k}^{2} \delta \alpha+\mathrm{k} \alpha-3 \lambda \mathrm{k} \delta \alpha^{2}=0$ which gives

$\alpha=\frac{1}{3}\left[\frac{\lambda^{2}}{\mathrm{k}^{3}}-\frac{1}{\delta}\right]$. Substitute values of $\mu, \alpha$ and $\beta$ into equation (38), we obtain

$\mathrm{u}(\mathrm{x}, \mathrm{y}, \mathrm{t})=\frac{1}{3}\left[\frac{\lambda^{2}}{\mathrm{k}^{3}}-\frac{1}{\delta}\right] \operatorname{Cot}\left( \pm\left[\frac{\lambda^{2}}{2 \mathrm{k}^{4}}-\frac{1}{2 \mathrm{k} \delta}\right]^{\frac{1}{2}} \xi\right)$

where $\xi=\mathrm{kx}+\delta \mathrm{y}+\lambda \mathrm{t}$.

And also using equations (33), (38) and $\mathrm{v}=-\frac{\delta \mathrm{u}^{2}}{2 \mathrm{k}}-\frac{\delta \lambda \mathrm{u}}{\mathrm{k}^{2}}$, we have 
$\mathrm{v}(\mathrm{x}, \mathrm{y}, \mathrm{t})=\frac{\delta}{18 \mathrm{k}}\left[\frac{\lambda^{2}}{\mathrm{k}^{3}}-\frac{1}{\delta}\right]^{2} \operatorname{Cot}^{2}\left( \pm\left[\frac{\lambda^{2}}{2 \mathrm{k}^{4}}-\frac{1}{2 \mathrm{k} \delta}\right]^{\frac{1}{2}} \xi\right)-\frac{\delta \lambda}{3 \mathrm{k}^{2}}\left[\frac{\lambda^{2}}{\mathrm{k}^{3}}-\frac{1}{\delta}\right] \operatorname{Cot}\left( \pm\left[\frac{\lambda^{2}}{2 \mathrm{k}^{4}}-\frac{1}{2 \mathrm{k} \delta}\right]^{\frac{1}{2}} \xi\right) \ldots$

where $\xi=\mathrm{kx}+\delta \mathrm{y}+\lambda \mathrm{t}$

\subsubsection{The Korteweg-dVries(Kdv) Equation as discussed by Arfken and Weber[3]}

The nonlinear Kuortweg-dVries equation, models the lossless propagation of shallow water waves and other phenomena. It is as follows:

$\Psi_{\mathrm{t}}+\Psi \Psi_{\mathrm{x}}+\Psi_{\mathrm{xxx}}=0$.

Again, we use the transformations.

$\Psi(\mathrm{x}, \mathrm{t})=\Psi(\xi), \xi=\mathrm{x}-\lambda \mathrm{t}$ with equation (8) to transform (43) to the linear ordinary

differential equation

$\mathrm{k}^{3} \Psi^{\prime \prime}+\mathrm{k} \Psi^{2}-\lambda \Psi=0$

Integrating (44) once, we obtain,

$\mathrm{k}^{3} \Psi^{\prime \prime}+\mathrm{k} \Psi^{2}-\lambda \Psi=0$

We seek the solution of $(45)$ as

$\Psi(\xi)=\alpha \tan ^{\beta}(\mu \xi)$, where

$\Psi^{\prime \prime}=\alpha \beta \mu^{2}\left[(\beta-1) \tan ^{\beta-2}(\mu \xi)+2 \beta \tan ^{\beta}(\mu \xi)+(\beta+1) \tan ^{\beta+2}(\mu \xi)\right]$

and let the expression of $\Psi$, and $\Psi^{\prime \prime}$ be represented by equation (46).

Substituting equation (46) into (45) and simplify, we have

$\mathrm{k}^{3} \alpha \beta \mu^{2}\left[(\beta-1) \tan ^{\beta-2}(\mu \xi)+2 \beta \tan ^{\beta}(\mu \xi)+(\beta+1) \tan ^{\beta+2}(\mu \xi)\right]+\mathrm{k}^{2} \tan ^{2 \beta}(\mu \xi)-\lambda \alpha \tan ^{\beta}(\mu \xi)=$

0 .

Equating the exponents of each pair of the tan function, we get

$\beta+2=2 \beta \Rightarrow \beta=2$

Substituting $\beta=2$ into equation (47) and simplify, we have

$2 \mathrm{k}^{3} \alpha \mu^{2}\left[4 \mathrm{k}^{3} \alpha \mu^{2}-\lambda \alpha\right] \tan ^{2}(\mu \xi)+\left[3 \mathrm{k}^{3} \alpha \mu^{2}+\mathrm{k} \alpha^{2}\right] \tan ^{4}(\mu \xi)=0$

Equating coefficients of each $\tan ^{\mathrm{k}}(\mu \xi)$ to zero, we get for $\tan ^{2}(\mu \xi)$,

$4 \mathrm{k}^{3} \alpha \mu^{2}-\lambda \alpha=0$

$\Rightarrow \mu=\frac{ \pm \sqrt{\lambda}}{2 \mathrm{k}^{2 / 3}}$ and for $\tan ^{4}(\mu \xi)$

$3 \mathrm{k}^{3} \alpha \mu^{2}+\mathrm{k} \alpha^{2}=0$

$\Longrightarrow \alpha=\frac{-3 \lambda}{4 \mathrm{k}}$

Substituting values of $\mu, \beta$ and $\alpha$ into equation (46), we get

$\Psi(\mathrm{x}, \mathrm{t})=-3 \lambda \tan ^{2}\left(\frac{-\lambda^{1 / 2}}{2 \mathrm{k}^{3 / 2}} \xi\right)$.

Where $\xi=\mathrm{x}-\lambda \mathrm{t}$.

\section{Results/ Discussion}

The new Tan-Cot Function Method has been applied successfully in this paper, to establish new solitary wave solutions for coupled and non coupled non linear partial differential equations.

However, the new method could not establish exact solutions for the coupled nonlinear partial differential momentum equations of Navier-Stokes' and the convection heat transfer equation, because the linear parts of these nonlinear partial differential equations cannot be separated from the nonlinear parts

\section{Conclusion}

We can therefore say that the new method can be extended to solve the problems of nonlinear partial differential equations which may arise in the theory of solitons and other area of nonlinear science.

\section{References}

[1]. Mahmood Jawal Abdul Rasool Abu Al-Shaeer. "Solutions for Nonlinear Partial Differential Equations by Tan-Cot Method". IOSR Journal of Mathematics, vol 5, Issue 3(Jan-Feb 2013), pp 06-11.

[2]. Anwar Ja'afar Mohammed Jawal. "New exact solutions of Nonlinear Partial Differential Equations Using Tan-Cot Function Method". Studies in Mathematical sciences, Vol.5, No 2, 2012, pp $13-25$

[3]. Nasir Taghizadeh et al. "Exact Traveling Wave solutions of the Coupled Klein-Gordon Equation by the infinite series method". Applications and Applied Mathematics, Vol 6, Issue 1 (June 2011), pp23-231

[4]. N. Taghizadeh et al. "Soliton Solutions of the Huxley equation by the modified-Expansion Method" Mathematica

[5]. Ram Daya Panka \& Arun Kumar, "Solutions of the Coupled Klein-Gordon Equation by Modified Exp-Function Method". Indian Journal of Applied Research,Research Paper, Mathematics, Vol: 3/ Issue: 3/ March 2013/ ISSN-2249-555X 
[6]. Anwar Ja'far Mohammed Jawal. "The solition solutions of nonlinear system (2+1)Dimensional Equations". IOSR Journal of Mathematics, Vol. 1, issue 6(July-Aug 2012), pp27-34

[7]. George B. Arfken \& Hans J. Weber, "Mathematical Methods for Physicists, sixth edition", pp 542

\section{Disclosure Policy}

We, the authors of "Exact Solution of Nonlinear Partial Differential Equations Using the New Tan-Cot Method" declare that there is no conflict of interests regarding the publication of this article. 